\title{
Cause of Neonatal Sepsis: Transplacental vs Environmental
}

\author{
Gauri N Bapat, Swati Shiradkar, Manjushree H Bhalchandra, Shubhangi Mande, Mangala Bansal
}

\begin{abstract}
Objectives: To study the role of transplacental transmission and environmental factors in neonatal sepsis (0-3) days and incidence of early neonatal sepsis in obstetrics and gynecology wards of MGM Medical College Hospital.
\end{abstract}

Materials and methods: The study was carried out on 100 full term women and their babies who delivered in MGM hospital. Their cord blood cultures were done. The nasal carrier state for Staphylococcus aureus ( $S$. aureus) in all the health professionals working in labor room was also studied.

Results: S. aureus was the most common organism grown in cord cultures of woman who delivered vaginally. None of the babies followed in wards showed any Sign of sepsis in first 72 hours. More than $50 \%$ of health professionals working in labor room were carriers of $S$. aureus.

Conclusion: S. aureus is having environmental source and it causes late neonatal sepsis so none of the babies developed any Sign of sepsis in first 72 hours of life. Other organisms isolated probably responded to intrapartum antibiotics or maternal immunity played its role and so babies did not showed any s/o sepsis in first 3 days.

Keywords: Neonatal sepsis, Cord blood culture, Nasal carrier.

How to cite this article: Bapat GN, Shiradkar S, Bhalchandra $\mathrm{MH}$, Mande S, Bansal M. Cause of Neonatal Sepsis: Transplacental vs Environmental. J South Asian Feder Obst Gynae 2013;5(3):132-134.

\section{Source of support: Nil}

Conflict of interest: None declared

\section{INTRODUCTION}

There is a vast difference in infant mortality rates (IMR) in developed and developing countries. In developed countries IMR is 5 to $7 / 1000$ live births, when their birth rate is 12 to $14 / 1000$ population. But in developing countries IMR is ten times more, i.e. 50 to 70/1000 live births when their birth rate is just double, i.e. 23 to 25 per 1000 population. The major bulk of this infant mortality rate is due to sepsis. The incidence of neonatal sepsis in developing countries is 7 to $38 / 1000$ live births and the most critical period is 0 to 3 days of life. Home deliveries account for $75 \%$ of these sepsis deaths but remaining is with hospital deliveries. Early identification of sepsis is essential to prevent these deaths. Umbilical cord blood culture studies are being tried since last 30 years for this purpose. ${ }^{1}$

\section{MATERIALS AND METHODS}

The study was carried out on 100 full-term women with their babies who delivered in our hospital. Selection was done randomly and the study period was 6 months from June 2011 to November 2011.

At the time of delivery, two clamps were applied at the placental and baby's side (Fig. 1). A piece of cord was cut in between the pair of clamps and was handed over to the assistant. The cord was wiped three times with 70\% isopropyl alcohol using sterile technique and around $2 \mathrm{ml}$ blood is collected from either umbilical vein or artery (Fig. 2). The needle was changed with a new sterile needle. The broth bottle top was cleaned with alcohol and blood was injected into broth ${ }^{2,3}$ (Fig. 3). The blood was incubated in blood culture bottles containing $10 \mathrm{ml}$ of brain heart infusion broth (BHIB) from Hi Media. These bottles were incubated at $37^{\circ} \mathrm{C}$ temperature under aerobic conditions. Subculture was done on Sheep Blood Agar and MaConkey's agar after 24, 48, 72, 96 hours. A provisional report was issued after every subculture and if after 96 hours no growth was obtained, it was reported negative. If any growth obtained, it was processed as per standard microbiological techniques. ${ }^{4}$ Antimicrobial sensitivity testing was performed by Kirby Bauer disk diffusion method. For antibiotic sensitivity testing commercially available disks from Hi Media were used and S. aureus (ATCC 25923), E. coli (ATCC 25922) were the strains used for quality control.

The babies were followed in the wards for 72 hours for any signs of neonatal sepsis like respiratory distress, refusal of feed, irritability, lethargy hyperthermia, pustules or abscesses, vomiting and abdominal distension.

Association of high risk factors in the mother like premature rupture of membranes, prolonged labor, multiple pelvic examinations, anemia, intrapartum fever, history of febrile illness in last 7 days and meconium stained liquor was also studied. ${ }^{5}$

Simultaneously, the nasal swabs of all the health professionals working in labor room, who were involved in patient's care were collected and cultured to assess the carrier state of $S$. aureus. ${ }^{6}$

\section{OBSERVATION}

Out of 100 women included in the study, 65 had vaginal delivery and 35 underwent cesarean section. Total 37 cord

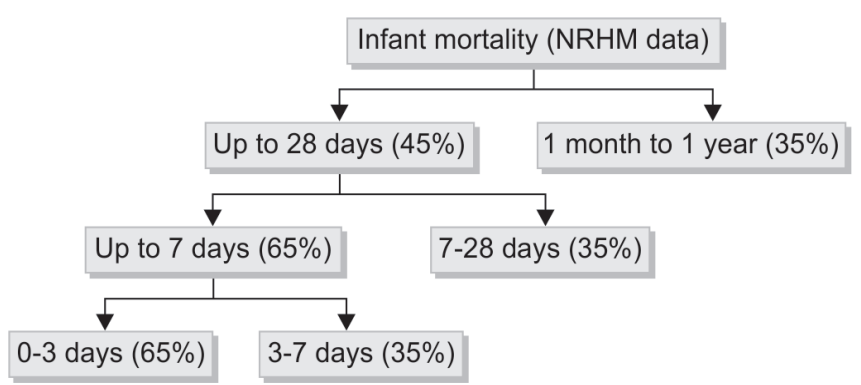




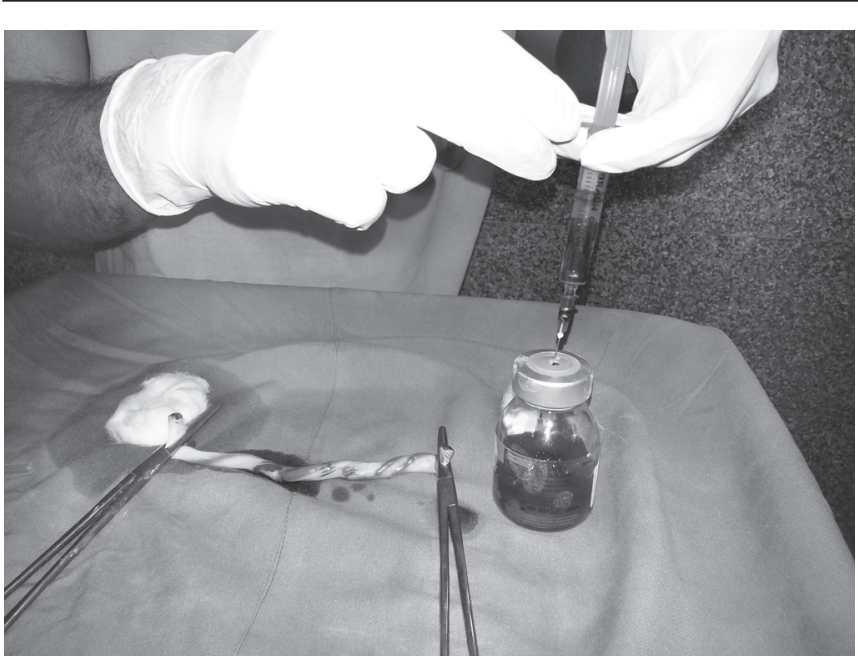

Fig. 1: A piece of cord is cut between two clamps

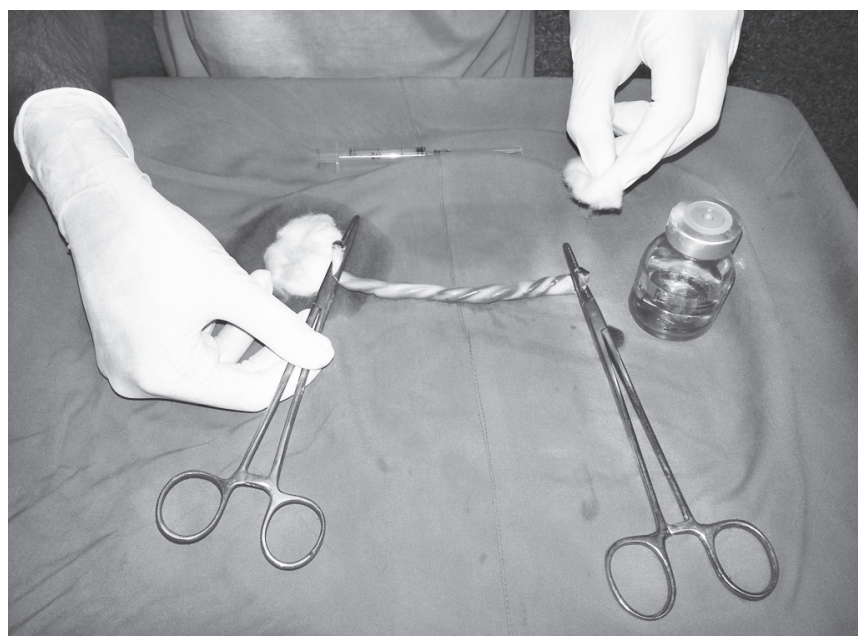

Fig. 2: The cord is cleaned with $70 \%$ isopropyl alcohol and $2 \mathrm{ml}$ blood withdrawn

Table 1: Correlation of high risk factors with positive cord blood cultures

\begin{tabular}{lccc}
\hline & High-risk factor & $\begin{array}{c}\text { +ve cord } \\
\text { blood culture }\end{array}$ & Incidence (\%) \\
\hline Present & 22 & 7 & $31.8 \%$ \\
Absent & 78 & 30 & $38.4 \%$ \\
\hline
\end{tabular}

\begin{tabular}{lccc}
\multicolumn{4}{c}{ Table 2: Correlation of antibiotics with positive } \\
cord blood cultures
\end{tabular}

blood cultures, out of 100, were positive. The incidence of positive cord blood cultures was more in vaginal delivery group, i.e. $53.7 \%$ as compared to cesarean section group, i.e. 5.7\%.

The list of organisms grown was topped by $S$. aureus (23) followed by E. coli and Enterococci (6 each) and other Gramnegative bacilli (2).

Antibiotic sensitivity pattern revealed that these organisms were sensitive to penicillins, cephalosporins, macrolides and

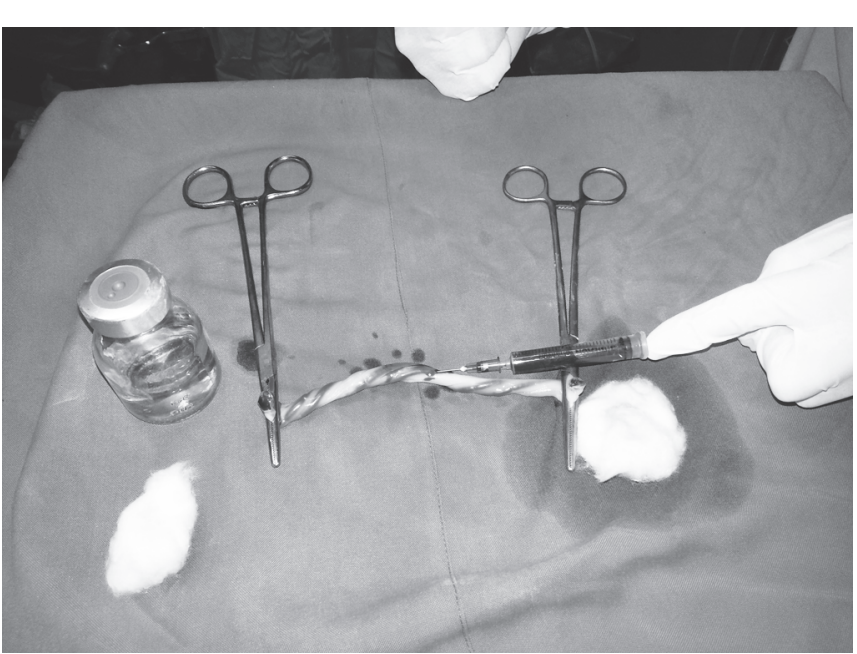

Fig. 3: Needle changed and blood is injected into the broth

aminoglycosides but were resistant to fluroquinolones and cotrimoxazole. $^{7}$

When we compared the presence and absence of high-risk factors (Table 1) and receiving or not receiving intrapartum antibiotics (Table 2) in the positive cultures, the incidences were almost same.

We could follow 91 babies in ward as 9 babies were shifted to NICU for varied reasons. None of the babies followed in the ward developed any signs of neonatal sepsis in 72 hours.

Out of 91 babies followed, 34 babies had positive cord blood cultures and 57 had negative cultures.

Nasal swabs of 36 persons were cultured, 21 of them had positive nasal cultures for $S$. aureus.

\section{DISCUSSION}

Neonatal sepsis is divided into early and late. Late sepsis is caused by $S$. aureus and coagulase negative staphylococci mainly and are transmitted from care giving environment at and after birth. Antepartum intrauterine infections and transplacental transmission is considered a major cause for early neonatal sepsis and group B streptococci, E. coli and coagulase negative staphylococci are the causative organisms mainly. ${ }^{8}$ As per Hansan et al (2005) and Ottolini et al (2003) umbilical cord blood culture is an effective method to diagnose neonatal sepsis. ${ }^{9-11}$ Our study of cord blood cultures could not prove the role of transplacental transmission of infection as the organism isolated in $62 \%$ of cases was $S$. aureus. This $S$. aureus proved to be an environmental pathogen because in $91 \%$ of babies there were no signs of sepsis in first 72 hours of life and $58 \%$ of health professionals were nasal carriers of $S$. aureus. The similar incidence of positive cultures in presence and absences of high risk factors and receiving or not receiving intrapartum antibiotics can also be explained on the same basis that the organism isolated in cultures had an environmental source. All other organisms isolated were sensitive to cephalosporins, which is used as intrapartum prophylaxis in our hospital. None of the babies, followed in wards either with positive cultures (34) or with negative cultures (57) developed any signs of sepsis in first 72 hours. 
S. aureus thus proves to be an environmental pathogen and the incidence of its nasal carrier state was proved to be $58 \%$ in our study so aggressive treatment of it was considered necessary. Nasal application of mupirocin (Bactroban) ointment twice a day for 7 days was advised to all carriers.

WHO recommends 5 ' $\mathrm{C}$ ' for the prevention of sepsis at the time of delivery which includes clean hands and fingernails, clean surface of delivery, clean cutting and care of cord, clean birth canal and clean environment. All of these are followed meticulously in hospitals except the clean environment. Our study highlighted the role of human pathogens and their sources from carriers, especially in overcrowded labor rooms of tertiary care hospitals. The carrier state of $S$. aureus and its contribution in hospital-acquired infections is to be considered seriously. Regular health check-up for carrier state and its treatment is essential as this has very high recurrence rate. Use of nasal masks by everyone giving care to a laboring patient and the newborn can be helpful in decreasing the incidence of neonatal sepsis. Follow-up of the babies after 7 days, i.e. first postpartum visit is very essential as these organisms can lead to late neonatal sepsis (i.e. upto 7 days) which can be missed till it becomes late. These simple measures are easy to follow and will definitely help us in decreasing neonatal mortality in first 7 days of life. Further studies are warranted to substantiate the exact role of this isolated $S$. aureus in neonatal sepsis which can be achieved with follow-up blood cultures of babies on 7 th day.

\section{REFERENCES}

1. Prolin JI, Knox SI, Bougngard S. Use of umbilical cord blood culture for detection of neonatal bacteremia. Obstet Gynecol 1981;57(2):233-237.

2. Costakos DT, Jennifer W, Mary R, Lynn D. Painless blood testing to prevent neonatal sepsis. Wisconsin Medical Journal 2009;108(6):321-322.

3. Farraj AA. Randomized placental and cord blood sampling culture in woman with preterm and term labour to detect infection. Eastern Mediterranean Health Journal 2000; $16(2 / 3): 272-275$

4. Vinodkumar CS, Neelagund YF. Incubation period for culture positive to detect septicemia in neonates. Indian J Med Microbiol 2005;23:270-271.

5. Hansen A, Forbes P, Buck R. Potential substitution of cord blood for infant blood in the neonatal sepsis evaluation. Biolneonate; $2005 ; 88(1)$
6. Klutmans J. Nasal carriage of S. aureus: Epidemiology, underlying mechanisms associated risk. Clinical Microbiology 1997;10(3):505-520.

7. Issacs D, fraser S, Hogg G. S. aureus infections in Australian neonatal nurseries. ADC Fetal and Neonatal edition 2004;89: F331-335.

8. Mahmood A, Karant KA, Butt T. Neonatal Sepsis-high antibiotic resistance of the bacterial pathogen in a neonatal intensive care unit in Karachi. J Pak Med Assoc 2002 Aug;52(8):348-350.

9. Ottolini, Mary C, et al. Utility of complete blood count and blood culture screening to diagnose neonatal sepsis in the asymptomatic at risk newborn. The Paediatric Infectious Disease Journal 2003;22(5):430-434.

10. Beeram R, Madhav S, Cheryl L, Cheryl C, Vinayak G. Utilization of umbilical cord blood for evaluation of Group B Streptococcus sepsis screening. Clinical Paediatrics. Cpj.sagepub.com/content/early/2011.

11. Janie A. Umbilical cord blood as an alternative for infant blood in the neonatal sepsis evaluation, Philosophy of Nursing Science, Texas Woman's University College of Nursing, Dallas center. Aug 11, 2009.

\section{ABOUT THE AUTHORS}

\section{Gauri N Bapat (Corresponding Author)}

Associate Professor, Department of Obstetrics and Gynecology Mahatma Gandhi Mission's Medical College, Aurangabad, Maharashtra India, Phone: 9890559047, e-mail: gaurimvaishnav@gmail.com

\section{Swati Shiradkar}

Professor and Head, Department of Obstetrics and Gynecology, Mahatma Gandhi Mission's Medical College, Aurangabad, Maharashtra, India

\section{Manjushree H Bhalchandra}

Associate Professor, Department of Microbiology, Mahatma Gandhi Mission's Medical College, Aurangabad, Maharashtra, India

\section{Shubhangi Mande}

Associate Professor, Department of Obstetrics and Gynecology, Mahatma Gandhi Mission's Medical College, Aurangabad, Maharashtra, India

\section{Mangala Bansal}

Professor and Head, Department of Microbiology, Mahatma Gandhi Mission's Medical College, Aurangabad, Maharashtra, India 